\title{
Surgical management of atrial fibrillation at the time of septal myectomy
}

\author{
Eduard Quintana ${ }^{1}$, James L. Cox ${ }^{2}$ \\ ${ }^{1}$ Cardiovascular Surgery Department, Institut Clínic Cardiovascular, Hospital Clínic de Barcelona, University of Barcelona Medical School, \\ Barcelona, Spain; ${ }^{2}$ Bluhm Cardiovascular Institute, Feinberg School of Medicine, Northwestern University Medical Center, Chicago, USA \\ Correspondence to: Eduard Quintana, MD, PhD, FETCS. Cardiovascular Surgery Department, Institut Clínic Cardiovascular, Hospital Clínic de \\ Barcelona, University of Barcelona Medical School, Wing 10, 3rd floor, Cardiovascular Surgery Intensive Care Unit, C/Villarroel 170 CP 08036, \\ Barcelona, Spain. Email: equintan@clinic.cat.
}

\begin{abstract}
Atrial fibrillation (AF) may appear during the natural clinical course of hypertrophic cardiomyopathy (HCM). It is regarded as a complication of HCM and is a marker of advanced disease. AF is more likely to occur in untreated, obstructive HCM. Unfortunately, this represents a turning point that puts patients at increased risk of further disability, stroke and death. The presence of obstruction is an indication to proceed with septal myectomy to improve or resolve symptoms by ameliorating diastolic dysfunction. If the patient has concomitant AF, a Maze procedure should be added to the septal myectomy. This combined operation yields excellent hemodynamic benefits and potentially decreases the risk of thromboembolic events.
\end{abstract}

Keywords: Hypertrophic cardiomyopathy (HCM); septal myectomy; atrial fibrillation (AF); surgical ablation; Maze

Submitted Mar 29, 2017. Accepted for publication Apr 07, 2017.

doi: $10.21037 /$ acs.2017.05.08

View this article at: http://dx.doi.org/10.21037/acs.2017.05.08

\section{Introduction}

Mortality in hypertrophic cardiomyopathy (HCM) results from unpredictable sudden cardiac death, heart failure, or stroke related to the presence of atrial fibrillation (AF). Symptoms in this patient population can arise from the presence of obstructive physiology, diastolic dysfunction due to restrictive physiology and/or AF that worsens global hemodynamic performance.

AF may occur as a consequence of longstanding diastolic heart failure secondary to increased left atrial pressure arising from the obstruction of the left ventricular outflow tract (LVOT). This results in varying degrees of associated secondary mitral regurgitation (i.e., AF secondary to structural heart disease). Additional potential reasons for developing AF are atrial sarcomeric myopathy or acquired risk factors such as hypertension (1).

In other HCM patients, AF may result from severe diastolic dysfunction inherent to restrictive physiology. Advanced restrictive HCM patients without obstruction constitute a very challenging group of patients that will not be discussed further.

The focus of attention when dealing with hypertrophic obstructive cardiomyopathy (HOCM) has been to treat medically, or to address the LVOT obstruction invasively in hopes of preventing sudden death. AF is the most common sustained arrhythmia in HCM, with a prevalence as high as $20 \%(1)$.

The development of AF represents a critical clinical turning point in HCM, impacting both the quality of life and mortality. More severe and disabling symptoms usually occur in HCM patients with the onset of AF. Regardless of the type of AF (paroxysmal, persistent, long-standing persistent or permanent AF), oral anticoagulation is indicated. Nevertheless, there are limited data to support either catheter ablation or surgical intervention for the treatment of AF in the HCM population. It is clear, however, that much like the experience with lone AF, catheter ablation for AF in patients with HCM has been suboptimal (2). For patients undergoing septal myectomy, a Maze procedure is supported with a Class IIa recommendation with a level of 
evidence $\mathrm{C}$ based on currently available guidelines (3).

\section{Clinical outcomes associated with AF and HCM}

Ischemic strokes occur almost eight times more frequently in AF-HCM patients than in patients with HCM who are in sinus rhythm, regardless of the AF type. The mortality rate for stroke in AF-HCM patients is $20 \%$ (4). Because the development of AF in patients with $\mathrm{HCM}$ is associated with a high risk of early death, oral anticoagulation is indicated even after only one episode, regardless of the $\mathrm{CHA}_{2} \mathrm{DS}_{2}$ VASc score, which has not been validated in this population (4-6). A $24 \mathrm{hr}$ Holter monitor is indicated every 6-12 months in patients with left atrial diameters (LADs) $>45 \mathrm{~mm}$, to screen for $\mathrm{AF}$ and define the risk of sudden death (Class IIA) $(3,7)$.

\section{The Gold Standard surgical arrhythmia procedure for lone AF}

The Maze III (which includes both the cut-and-sew Maze III and the Minimally Invasive Cryo-Maze III) was designed to abort or block all potential macro re-entrant circuits in both atria that sustain AF. The operation itself proved to be successful in preventing long-term recurrences (median follow up of 5.9 years) by current definitions of success in $83 \%$ of patients. Those patients had a preoperative median duration of AF of 7 years. Freedom from symptomatic AF at 10 years was $85 \%$.

It is important to note that those results were achieved in a population that presented with a mean left atrial diameter (LAD) of $47 \mathrm{~mm}$ and only $60 \%$ had paroxysmal AF (8). Long-term thromboembolic complications (stroke/TIA) following the Maze III procedure occurred in only 1 of 112 patients $(0.8 \%)$, despite the fact that $86 \%$ of the patients were not anticoagulated.

Despite the demonstrated efficacy of this approach, the operation has not been widely adopted because of its perceived complexity and it is currently performed only by a relatively small number of surgeons. A more appealing and faster surgical approach to perform what is essentially the same lesion set, was introduced by Damiano's group in 2003 (9) with the use of bipolar radiofrequency and cryothermia, the (Maze IV). The potential for not achieving completely transmural lesions with the use of this primarily radiofrequency approach, may result in less overall success $(66 \%$ success at 5 y) (10) than with the classic cut-andsew or completely cryosurgical Maze III procedure.
Certainly, when other minimalistic arrhythmia options (e.g., radiofrequency pulmonary vein isolation) are used to treat both PAF and non-PAF, the observed outcomes are less successful (65\% at 1 year) (11).

\section{Extrapolation of a Maze operation to concomitant cardiac surgery and HCM}

Unfortunately, the results of AF surgical ablation for AF associated with hypertrophic obstructive cardiomyopathy are limited (12).

$\mathrm{AF}$, in the setting of any structural heart defect amenable to surgical correction, may represent a different disease because rather than the AF appearing de-novo, it is commonly the result of left-heart disease such as mitral valve, aortic valve, or ischemic heart disease, as well as systemic hypertension. HCM is in the group of aortic valve diseases, but its pathophysiology is markedly different from that of valvular aortic stenosis. Early success with concomitant $\mathrm{AF}$ ablation associated with non-HCM aortic valve disease and mitral valve disease is above $75 \%$ in the absence of the classical risk factors for poor prognosis (age beyond 75, LA Diameter over $50 \mathrm{~mm}$, duration of AF more than 5 years and non-paroxysmal AF) $(13,14)$. For concomitant AF ablative surgery, outcomes with the classic Maze III lesion pattern (either cut-and-sew or cryoablation) can be expected to be superior to those achieved with "modifications" of the original lesion set $(15,16)$. When using the original lesion pattern, the long-term success rate did not vary on the basis of the classic predictors of failure $(15,17)$.

Due to its simplicity, pulmonary vein isolation has remained an option to treat $\mathrm{AF}$ at the time of concomitant heart procedures. This approach, while often satisfactory for the treatment of PAF, yields far worse outcomes for non-PAF (persistent AF or long-standing persistent AF) than does a full biatrial procedure $(18,19)$.

Interpretation of the current literature can be confusing in the presence of so many permutations of the original biatrial Maze lesion pattern and the multiplicity of ablative energy sources. Yet, extrapolating current available retrospective studies to AF-HCM patients, one could expect excellent outcomes in those who undergo a proper Maze procedure and simultaneous relief of the LVOT obstruction.

\section{Rational for surgical ablation of AF at the time of septal myectomy}

A septal myectomy combined with a Maze III or Maze 
IV procedure has the potential benefit of restoring atrioventricular synchrony and improving atrial transport function, thereby yielding superior postoperative hemodynamics. Additionally, long-term outcomes may be improved by decreasing the risk of stroke, non-cerebral systemic embolic events and the need for additional medications or procedures.

Much like AF surgery, septal myectomy is an operation best performed by an experienced surgeon at a dedicated centre. Regardless of the presence of AF, the main goal of septal myectomy is to relief the LVOT obstruction completely with negligible morbidity and mortality. In this perspective article, we provide some insight into how to treat these complex and unusual patients based on our current practice and the lessons learned from the accumulating experience in treating lone and concomitant $\mathrm{AF}$ (11).

\section{Preoperative assessment}

All HCM patients have 12-lead ECG and $24 \mathrm{hr}$ Holter monitoring prior to the operation, to stratify the risk of sudden death and to detect any subclinical AF. Echocardiographic and magnetic resonance imaging provides data on atrial diameter and volume. The remaining work-up is that of any HCM patient. Interestingly, surface ECG alone may provide some prognostic value (Figure 1) to the procedure (20). Intraoperative transesophageal echocardiographic assessment is used to rule out the presence of thrombi inside the left atrial appendage.

\section{Proposed algorithm to deal with AF at the time of septal myectomy}

Resolution of LVOT obstruction is mandatory to ensure success of any AF ablation approach and this should be the first goal of the operation. A proposed schematic approach to help with decision-making in patients with AF and HCM is shown in Figure 2. Other important risk factors include age, degree of restrictive left ventricular physiology and scarring, hypertension, obesity, diabetes, duration of $\mathrm{AF}$, pulmonary hypertension, and associated heart valve dysfunction.

\section{Left atrial appendage (LAA) management}

Despite the evidence that concomitant amputation or occlusion of the LAA reduces the risk of thromboembolic events associated with $\mathrm{AF}$ (21-23) it remains unclear if the
LAA should be excluded in patients with AF and HCM.

The role of LAA management at the time of concomitant cardiac surgery will potentially clarify with the results of ongoing trials (LAOS III study).

It has been shown experimentally that with a normal right-sided cardiac output, the left ventricle adapts perfectly to the delivered blood flow through the pulmonary circulation if there is normal left ventricular function (24). Unfortunately, this physiology may not be relevant in patients with significant left ventricular diastolic dysfunction. Since only a proportion of HCM patients after myectomy have persistent LV diastolic dysfunction following a successful procedure, it may be safe to exclude the LAA in these patients. Unfortunately, that question has not been answered yet. This is an important issue because with $\mathrm{LV}$ diastolic dysfunction and a "stiff" left atrium, the LAA may be the only capacitance chamber on the left side of the heart and its removal could theoretically increase left atrial and pulmonary artery pressures postoperatively. In addition, preserving the LAA might enhance left atrial transport function during sinus rhythm postoperatively (25). On the other hand, if the patient has had previous thromboembolic events believed to be due to $\mathrm{AF}$, it would seem preferable to exclude the LAA unless the LV diastolic dysfunction is severe. It is our current policy to leave the appendage open in HCM patients when: (I) there have been no previous thromboembolic events and (II) if a residual, significant degree of diastolic dysfunction is expected regardless of a perfect septal myectomy. It is our belief that the appendage excision has potential benefits in terms of thromboembolic and arrhythmia risk reduction (26).

\section{Surgical technique}

It is our preferred approach to perform septal myectomy through a full median sternotomy because it allows control of all mediastinal structures and permits easy invasive left ventricle-aorta pressure measurements. Invasive gradient measurement is performed after aortic cannulation and before venous cannulation, to avoid atrial arrhythmias if the patient is in sinus rhythm. The superior vena cava (SVC) is cannulated high and the inferior vena cava (IVC) more medially than usual, to facilitate the right-sided Maze lesions. Hypothermic cardiopulmonary bypass is instituted at 32 degrees Celsius. Before arresting the heart, Sondergaard's groove is dissected free of fat to facilitate exposure and to ensure achievement of lesion transmurality. The right pulmonary veins are then encircled and isolated 
A

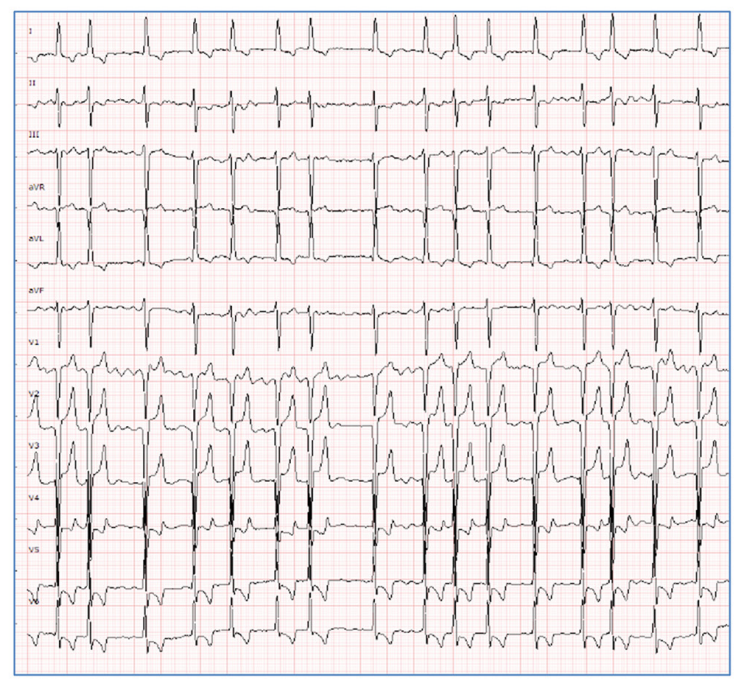

$\mathrm{C}$

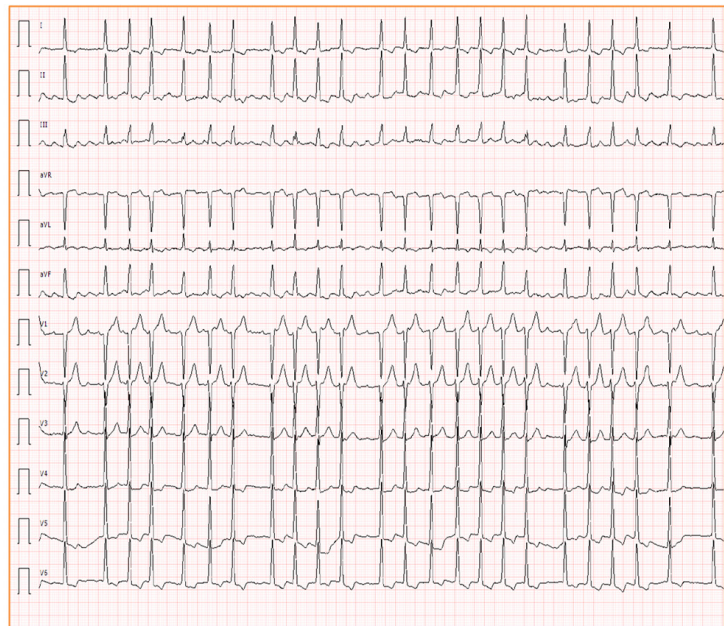

B

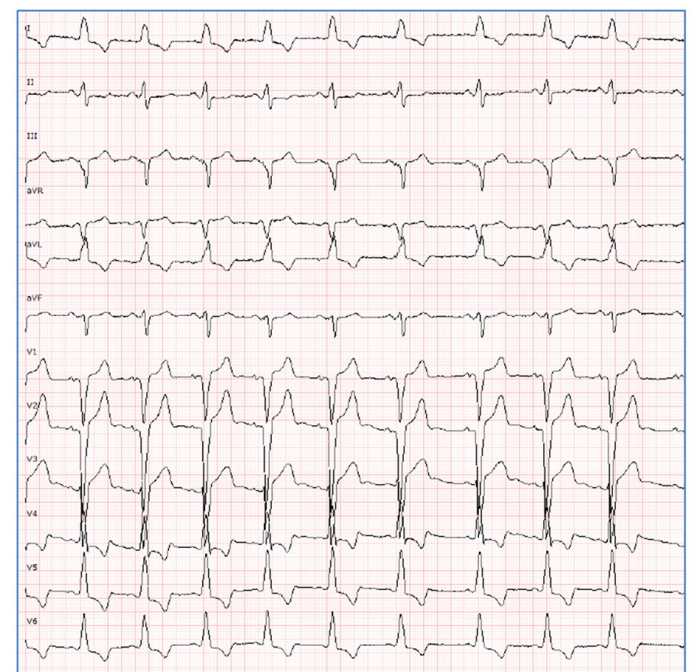

D

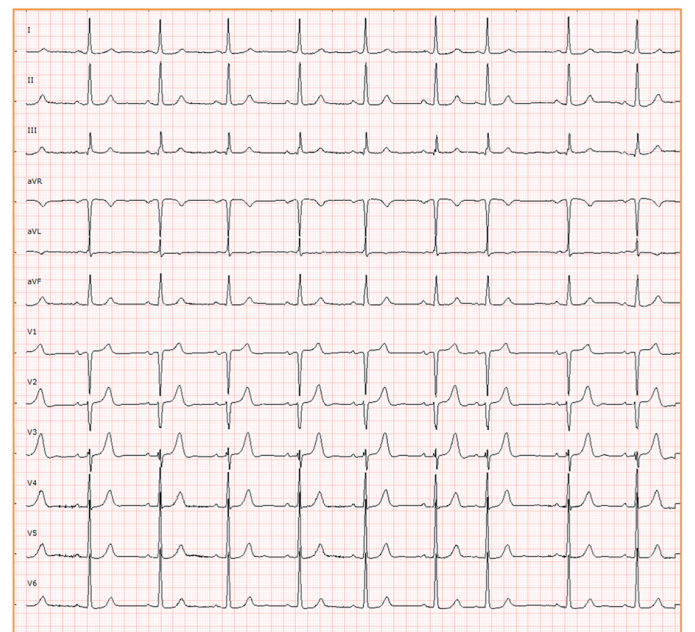

Figure 1 Preoperative and postoperative electrocardiography. (A,B) Preoperative and postoperative 12-lead surface electrocardiogram in a 71-year-old female with long standing persistent atrial fibrillation and severe LVOT obstruction with severe pulmonary hypertension undergoing the combined operation. An extended septal myectomy, Cox-Maze IV and tricuspid annuloplasty were performed. See the fibrillatory atrial waves in the preoperative ECG (upper left). On postoperative 6-month follow up sinus rhythm can be appreciated as well as the complete left bundle branch block after septal myectomy (upper right). (C,D) Preoperative and postoperative electrocardiogram of a 68y-o female patient with long standing persistent atrial fibrillation, severe LVOT obstruction and moderate aortic valve stenosis undergoing transaortic septal myectomy, Cox-Maze IV and tissue aortic valve replacement. Marked fibrillatory atrial waveforms can again be appreciated preoperatively (C). In this case, restoration of sinus rhythm can be appreciated but left bundle branch block did not occur (D). LVOT, left ventricular outflow tract.

well onto the left atrium away from their orifices using bipolar radiofrequency clamps. The heart is then arrested with antegrade blood cardioplegia, which is reinfused either antegrade or retrograde every $20 \mathrm{~min}$. Once the heart is arrested and emptied through a root vent, enucleation of the heart is achieved and the left pulmonary veins are isolated with bipolar radiofrequency, again being sure that the ablation lines are well onto the body of the left atrium. This is followed by surgical excision of the LAA and creation of another radiofrequency lesion from the open LAA, into the orifice of the left superior pulmonary vein. The LAA is closed with a double 4/0 polypropylene suture. At this stage, an aortotomy is performed, an extended septal myectomy is carried out and closure of the aorta subsequently follows. 
HOCM and atrial fibrillation surgical management algorithm

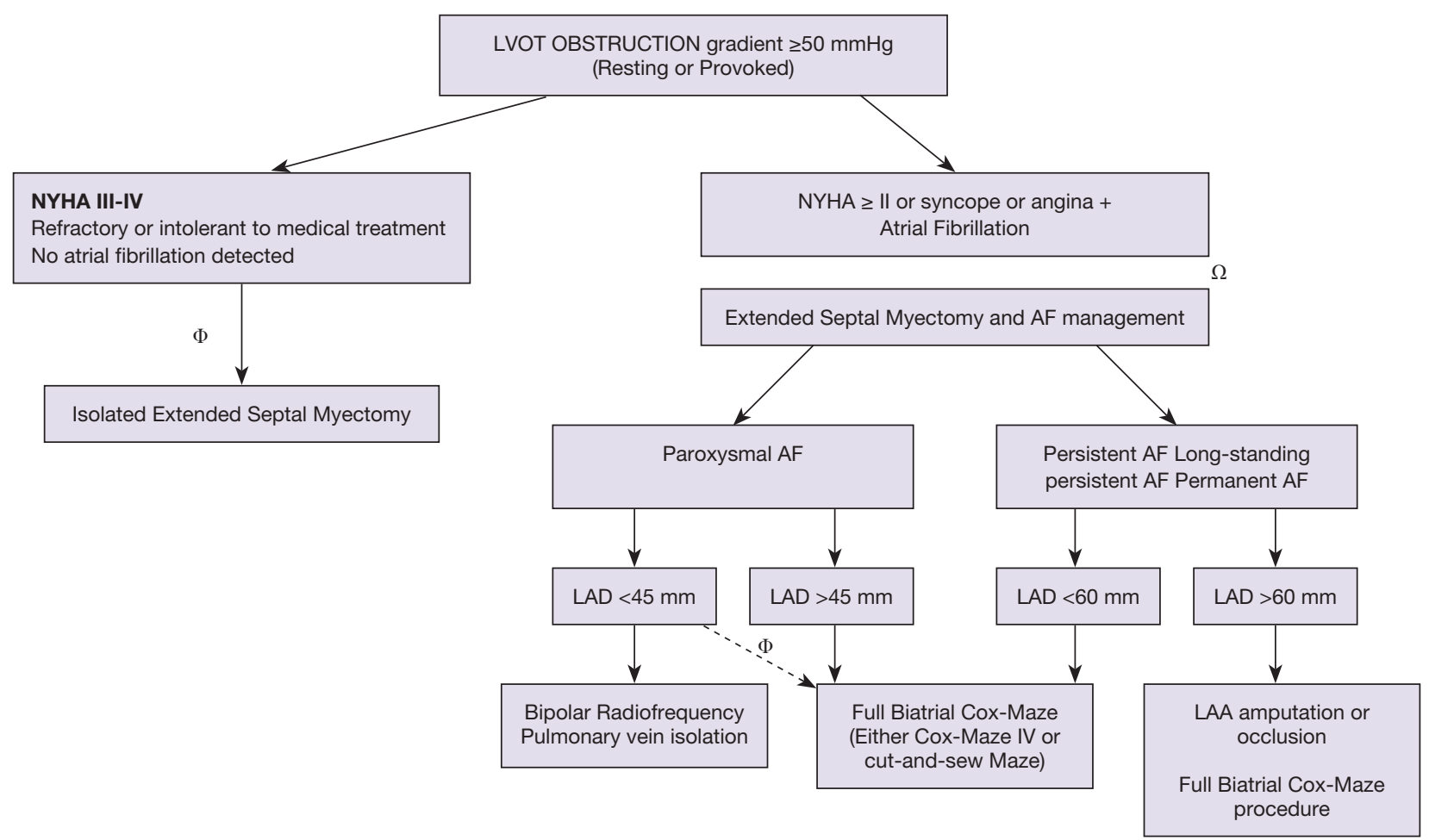

Figure 2 Proposed algorithm to treat patients with LVOT obstruction and AF. $\Phi$ currently accepted guideline management (AHA 2011, ESC 2014). $\Omega$, in experienced centres under the care of experienced operators the indications for such approach can be advanced if expected morbimortality is low. $\Phi$, in the presence of risk factors potential associated with poor outcomes of radiofrequency ablation such as age, hypertension, expected remaining significant diastolic dysfunction after myectomy. HOCM, hypertrophic obstructive cardiomyopathy; LVOT, left ventricular outflow tract; NYHA, New York Heart Association; AF, atrial fibrillation; LAD, left atrial diameter; LAA, left atrial appendage.

The left atrial Maze lesions are then completed by creating a "box" around all four pulmonary veins and the posterior wall of the left atrium, by placing both a roof lesion and a floor lesion between the superior pulmonary veins and inferior pulmonary veins respectively (Figure 3). An endocardial lesion from the left atriotomy towards the mitral valve is done with criothermy followed by an epicardial lesion at the same mitral valve plane that ablates the coronary sinus. Rewarming is started potentially at this point. The left atriotomy is closed and a $\mathrm{LV}$ vent is left in place. De-airing manoeuvres are performed and the aortic cross-clamp released.

It should be noted that in extremely hypertrophied left ventricles, enucleation of the heart and access to the left pulmonary veins may become challenging. If this is the case, completion of the left atrial Maze lesions and appendage line can be done from the endocardium with surgical cryoprobes and the LAA can be closed in two layers from within the left atrium. Usually, these lesions are placed following the myectomy procedure.

With the heart beating, the right atrial Maze lesions are performed. A horizontal right atriotomy is performed. A radiofrequency lesion is placed with the clamp up to the SVC through the atriotomy, followed by another lesion towards the IVC to complete the intracaval lesion between the SVC and the IVC (the IVC caval snare may be released to accommodate clamp placement). Another lesion is performed from the medial part of the atriotomy, close to the right auriculoventricular groove, to the right atrial appendage. The remaining lesion is done with criothermy from the tip of the atriotomy, down to the tricuspid annulus. The right atriotomy is closed, ventricular and atrial pacing 

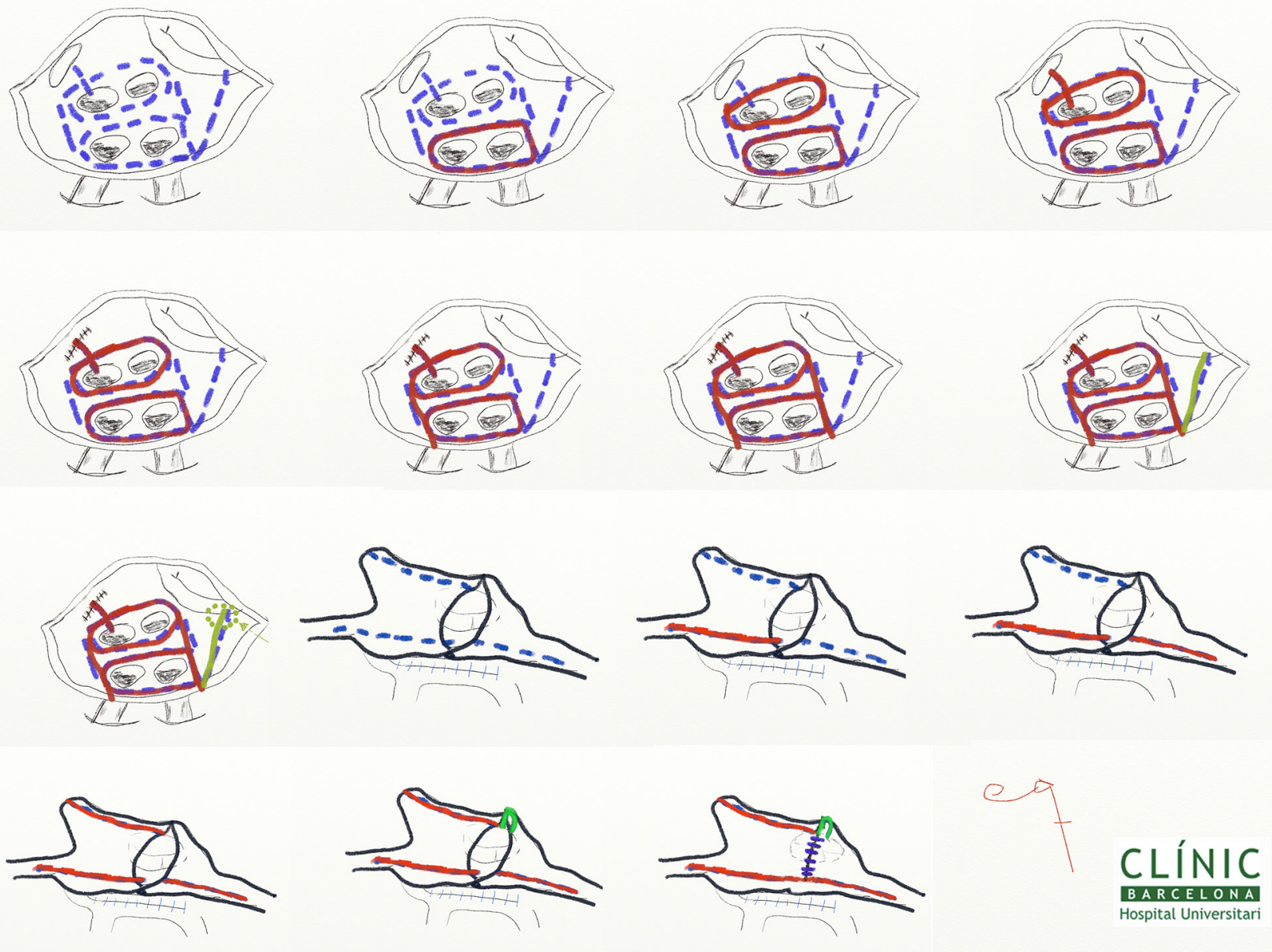

Figure 3 Cox-Maze IV surgical AF ablation scheme. In dashed blue lines the proposed lesion set for the Cox-Maze IV (biatrial Maze). In red those lesions performed with bipolar radiofrequency ablation. In green, lesions performed with cryothermia. Order of lesions from left upper corner to the right starting with the left sided Maze and followed by the right side. Green arrow shows the lesion performed epicardially to the coronary sinus. AF, atrial fibrillation.

electrodes are placed, cardiopulmonary bypass is weaned and invasive measurements are performed in resting and provoked conditions to ensure no residual gradients remain.

\section{Postoperative considerations. Anticoagulation. Long term follow up}

Beta-blockers are started as soon as it is felt to be safe after the operation, usually at $12-18$ hrs postoperatively. Regardless of the recurrence of early AF, anticoagulation is started postoperatively, usually on the $2^{\text {nd }}$ postoperative day with vitamin $\mathrm{K}$ antagonists and coverage with lowweight molecular heparin at full doses, if needed, until the INR approaches its target of 2.0-3.0. If AF occurs, postoperatively amiodarone is instituted and electrical cardioversion is employed if needed. Oral amiodarone is prescribed if postoperative AF has occurred and it is maintained for approximately one month based on rhythm. It is debatable whether anticoagulation withdrawal is appropriate but not supported by current guidelines and it should be judged in an individual basis (7) (Videos 1,2).

\section{Conclusions}

$\mathrm{AF}$ in HCM is a complex clinical problem and is usually a marker of advanced disease. $\mathrm{AF}$ in HOCM is frequent and 
leads to significant morbidity and mortality. Concomitant AF ablation surgery (Maze procedure) can be beneficial in patients undergoing septal myectomy, yielding excellent hemodynamic benefits and potentially decreasing the risk of thromboembolic events. This operation should be carried by experienced surgeons in both techniques.

\section{Acknowledgements}

To Manuel Castella, MD, PhD for establishing and developing the atrial fibrillation surgical ablation program at Hospital Clínic de Barcelona.

\section{Footnote}

Conflicts of Interest: The authors have no conflicts of interest to declare.

\section{References}

1. MacIntyre C, Lakdawala NK. Management of Atrial Fibrillation in Hypertrophic Cardiomyopathy. Circulation 2016;133:1901-5.

2. Gaita F, Di Donna P, Olivotto I, et al. Usefulness and safety of transcatheter ablation of atrial fibrillation in patients with hypertrophic cardiomyopathy. Am J Cardiol 2007;99:1575-81.

3. Gersh BJ, Maron BJ, Bonow RO, et al. 2011 ACCF/AHA guideline for the diagnosis and treatment of hypertrophic cardiomyopathy: a report of the American College of Cardiology Foundation/American Heart Association Task Force on Practice Guidelines. J Thorac Cardiovasc Surg 2011;142:e153-203.

4. Olivotto I, Cecchi F, Casey SA, et al. Impact of atrial fibrillation on the clinical course of hypertrophic cardiomyopathy. Circulation 2001;104:2517-24.

5. Maron BJ, Olivotto I, Bellone P, et al. Clinical profile of stroke in 900 patients with hypertrophic cardiomyopathy. J Am Coll Cardiol 2002;39:301-7.

6. Guttmann OP, Rahman MS, O'Mahony C, et al. Atrial fibrillation and thromboembolism in patients with hypertrophic cardiomyopathy: systematic review. Heart 2014;100:465-72.

7. Kirchhof P, Benussi S, Kotecha D, et al. 2016 ESC Guidelines for the management of atrial fibrillation developed in collaboration with EACTS. Eur J Cardiothorac Surg 2016;50:e1-e88.

8. Weimar T, Schena S, Bailey MS, et al. The cox-maze procedure for lone atrial fibrillation: a single-center experience over 2 decades. Circ Arrhythm Electrophysiol 2012;5:8-14.

9. Gaynor SL, Diodato MD, Prasad SM, et al. A prospective, single-center clinical trial of a modified Cox maze procedure with bipolar radiofrequency ablation. J Thorac Cardiovasc Surg 2004;128:535-42.

10. Henn MC, Lancaster TS, Miller JR, et al. Late outcomes after the Cox maze IV procedure for atrial fibrillation. J Thorac Cardiovasc Surg 2015;150:1168-76, 78.e1-2.

11. Boersma LV, Castella M, van Boven W, et al. Atrial fibrillation catheter ablation versus surgical ablation treatment (FAST): a 2-center randomized clinical trial. Circulation 2012;125:23-30.

12. Chen MS, McCarthy PM, Lever HM, et al. Effectiveness of atrial fibrillation surgery in patients with hypertrophic cardiomyopathy. Am J Cardiol 2004;93:373-5.

13. Ad N, Holmes SD, Lamont D, et al. Left-Sided Surgical Ablation for Patients With Atrial Fibrillation Who Are Undergoing Concomitant Cardiac Surgical Procedures. Ann Thorac Surg 2017;103:58-65.

14. Ad N, Holmes SD, Massimiano PS, et al. The effect of the Cox-maze procedure for atrial fibrillation concomitant to mitral and tricuspid valve surgery. J Thorac Cardiovasc Surg 2013;146:1426-34; discussion 34-5.

15. Albage A, Johansson B, Kenneback G, et al. Long-Term Follow-Up of Cardiac Rhythm in 320 Patients After the Cox-Maze III Procedure for Atrial Fibrillation. Ann Thorac Surg 2016;101:1443-9.

16. Philpott JM, Zemlin CW, Cox JL, et al. The ABLATE Trial: Safety and Efficacy of Cox Maze-IV Using a Bipolar Radiofrequency Ablation System. Ann Thorac Surg 2015;100:1541-6; discussion 7-8.

17. Prasad SM, Maniar HS, Camillo CJ, et al. The Cox maze III procedure for atrial fibrillation: long-term efficacy in patients undergoing lone versus concomitant procedures. J Thorac Cardiovasc Surg 2003;126:1822-8.

18. Henn MC, Lawrance CP, Sinn LA, et al. Effectiveness of Surgical Ablation in Patients With Atrial Fibrillation and Aortic Valve Disease. Ann Thorac Surg 2015;100:1253-9; discussion 9-60.

19. Pecha S, Schafer T, Subbotina I, et al. Rhythm outcome predictors after concomitant surgical ablation for atrial fibrillation: a 9-year, single-center experience. J Thorac Cardiovasc Surg 2014;148:428-33.

20. Nault I, Lellouche N, Matsuo S, et al. Clinical value of fibrillatory wave amplitude on surface ECG in patients with persistent atrial fibrillation. J Interv Card 
Electrophysiol 2009;26:11-9.

21. Cox JL, Ad N, Palazzo T. Impact of the maze procedure on the stroke rate in patients with atrial fibrillation. J Thorac Cardiovasc Surg 1999;118:833-40.

22. Holmes DR Jr, Kar S, Price MJ, et al. Prospective randomized evaluation of the Watchman Left Atrial Appendage Closure device in patients with atrial fibrillation versus long-term warfarin therapy: the PREVAIL trial. J Am Coll Cardiol 2014;64:1-12.

23. Reddy VY, Doshi SK, Sievert H, et al. Percutaneous left atrial appendage closure for stroke prophylaxis in patients with atrial fibrillation: 2.3-Year Follow-up of the PROTECT AF (Watchman Left Atrial Appendage System for Embolic Protection in Patients with Atrial Fibrillation) Trial. Circulation 2013;127:720-9.

24. Williams JM, Ungerleider RM, Lofland GK, et al. Left atrial isolation: new technique for the treatment of supraventricular arrhythmias. J Thorac Cardiovasc Surg 1980;80:373-80.

25. Isobe $F$, Kumano H, Ishikawa $T$, et al. A new procedure for chronic atrial fibrillation: bilateral appendage-preserving maze procedure. Ann Thorac Surg 2001;72:1473-8.

26. Di Biase L, Burkhardt JD, Mohanty P, et al. Left Atrial Appendage Isolation in Patients With Longstanding Persistent AF Undergoing Catheter Ablation: BELIEF Trial. J Am Coll Cardiol 2016;68:1929-40.

Cite this article as: Quintana E, Cox JL. Surgical management of atrial fibrillation at the time of septal myectomy. Ann Cardiothorac Surg 2017;6(4):386-393. doi: 10.21037/ acs.2017.05.08 